\title{
Reaching for atomic-scale quantitative energy dispersive X-ray spectroscopy
}

Katherine MacArthur ${ }^{1}$, Andrew Yankovich ${ }^{2}$, Armand Béché ${ }^{3}$, Martina Luysberg ${ }^{1}$, Hamish Brown ${ }^{4}$, Scott Findlay $^{5}$, Marc Heggen ${ }^{1}$ and Leslie Allen ${ }^{6}$

${ }^{1}$ Ernst Ruska Centre for Microscopy and Spectroscopy with Electrons and the Peter Grünberg Institute Forschungszentrum Jülich, 52425 Jülich, Germany, Germany, ${ }^{2}$ Department of Physics, Chalmers University, Sweden, ${ }^{3}$ Electron Microscopy for Materials Science (EMAT), University of Antwerp, 2020 Antwerp, Belgium, Belgium, ${ }^{4}$ The University of Melbourne, Parkville, Victoria, Australia, ${ }^{5}$ School of Physics and Astronomy, Monash University, Clayton, Victoria 3800, Australia, Clayton, Victoria, Australia, ${ }^{6}$ School of Physics, University of Melbourne, Parkville, Victoria 3010, Australia, Australia

The combined development of silicon drift detectors (SDDs) and aberration correction in the scanning transmission electron microscope (STEM) has a significantly advanced the field of microanalysis [1,2]. In particular, the combination of improved detector efficiency and high current densities really opens up the possibilities of quantitative energy dispersive X-ray (EDX) spectroscopy. We can now routinely achieve beautiful EDX maps which reveal atomic-scale features within our materials. However, it still remains to understand exactly where the X-ray signals come from within the specimen and how accurately they can be quantified at these length scales.

For atomic resolution imaging (when a crystal is necessarily aligned along a low order zone-axis), socalled electron channeling makes the beam-sample interactions non-linear and far harder to interpret [13]. Channeling occurs when columns of atoms aligned parallel to the electron beam provide an additional focusing effect on the electron beam, increasing the electron density close to the atomic nuclei and thereby producing a higher number of scattering and ionization events [3, 4]. For longer columns this focusing eventually produces a cross-over point within the sample which turns into oscillations of the probe electron density down the length of the column. This variation in probe electron density along atomic columns complicates composition measurements from the EDX signal not only due to the deviation from a linear relationship between atomic density and measured signal but also because it means the atomic ordering along the column can affect the signal.

Excellent quantitative agreement between calibrated experiments and multislice simulations using MuSTEM have been demonstrated [5, 6]. However, the challenge we often face in most interesting materials science applications is that we do not know the atomic-structure well enough to have something to simulate. Therefore, we need an approach that allows us to suppress the channeling and create a first guess of the materials structure using simple linear-based EDX quantification methods. Using $\mathrm{SrTiO}_{3}-$ $\mathrm{DyScO}_{3}$ multilayers as an example, we will discuss the combined effect of sample tilt [4] and spatial integration for ameliorating the effects of channeling and improving the accuracy of EDX quantification. Figure 1 shows the effect of sample tilt on integrated line profiles of the Ti-K line in both simulations and experiment. In suppressing channeling the peak to background ratio drops of significantly, however this brings the absolute values closer to the linear approximation [4]. Therefore, there is a balance to be found between beautiful atomic-scale but only qualitative maps and improving the quantification at a cost of some loss of resolution. Finally, we will also look at the effects of scattering and beam broadening and how this affects our ability to measure the accurate composition profile across an interface. The remaining 
limitations and the challenges will be discussed. In particular, the possibility for combining with 4DSTEM will be explored. [7]
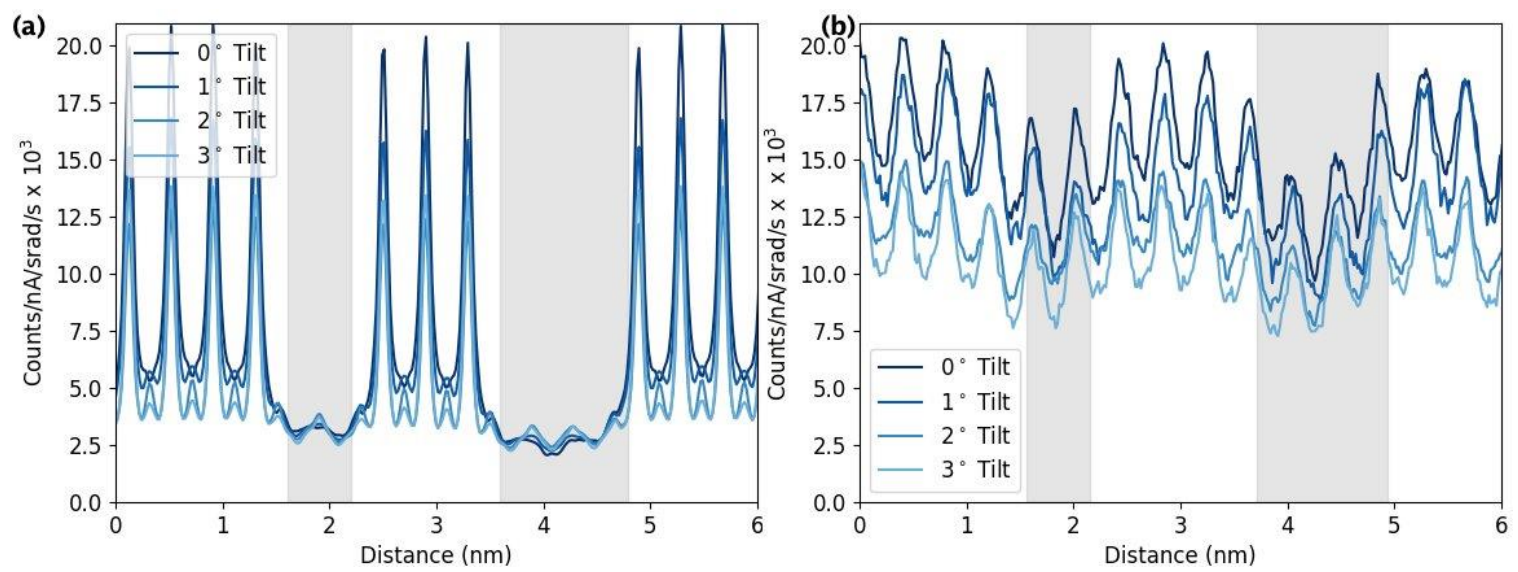

Figure 1. Comparison of quantified $\mathrm{X}$-ray line profiles for $\mathrm{Ti}-\mathrm{K}$ in a $\mathrm{SrTiO}_{3}-\mathrm{DyScO}_{3}$ multilayer oxide from simulation (a) and experiment (b) at different sample tilts, the grey regions indicate the approximate location of the $\mathrm{DyScO}_{3}$ layers. (a) is simulated with a perfectly abrupt interface. Although the match between experiment and simulation is close in average value there is still a significant resolution different which needs to be understood. (b) There is also no clear distinction between the layers in (b) although this is in part due to beam broadening and scattering occurring.

\section{References}

[1] P.J. Phillips et al. Microsc Microanal. 20 (2014), p. 1046-1052

[2] M. Watanabe et al. Microsc. Microanal. 12 (2006), p. 512-526

[3] K.E. MacArthur et al. Ultramic. 156 (2015), p. 1-8

[4] K.E. MacArthur et al. Ultramic. 182 (2016), p. 264-275

[5] Z. Chen et al. Ultramic. 168, (2016), p. 7-16

[6] N.R. Lugg et al. Ultramic. 151 (2015), p.150-159

[7] The authors acknowledge financial support from the DFG (grant number HE 7192/1-2), the Discovery Projects funding scheme of the Australian Research Council (Projects DP140102538 and FT190100619) and the European Union's Horizon 2020 research innovation program under grant agreement No. 823717 - ESTEEM3. 